\title{
Réflexions sur l'avenir de la thérapie génique
}

La thérapie génique, au même titre que le séquençage du génome humain, exprime I'adhésion implicite de notre génération à un postulat selon lequel I'amélioration de nos conditions de vie devrait passer par une meilleure connaissance des mécanismes du vivant. Après quinze ans de travail acharné, qu'ont apporté les recherches sur la thérapie génique? A quelles difficultés sommes nous confrontés? Comment imaginons-nous les développements futurs ? En bref, la thérapie génique pourra-t-elle vérifier ce postulat? Ce texte est le compte rendu d'un débat organisé autour de ces questions. Pour une description des travaux de thérapie génique eux-mêmes, les lecteurs pourront se référer au numéro thématique publié par médecine/sciences en mai 1999*.

\footnotetext{
* m/s n5, vol. 15 , mai 1999.
}

\section{Jean-Michel Heard Olivier Danos Marc Peschanski}

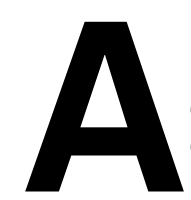
près un décollage précipité, la thérapie génique est rapidement entrée dans la zone de fortes turbulences que nous traversons actuellement. La navigation est sans visibilité, le cap éminemment variable et la destination imprécise, mais la machine est lancée, ses moteurs sont puissants et abondamment alimentés.

A partir d'une réflexion commune sur l'évolution de la discipline au cours des dernières années, nous avons essayé d'analyser les tendances qui ont orienté les développements de la thérapie génique et d'imaginer quelles directions pourraient être prises dans un futur proche ou plus lointain. L'exercice est bien évidemment périlleux, et nous sommes parfaitement conscients de ses limites et du ridicule auquel nous nous exposons si ce texte devait être relu dans quelques années. II nous a toutefois paru utile de le faire pour établir les données du débat telles qu'elles peu-
J.M. Heard : Laboratoire rétrovirus et transfert génétique, Cnrs URA 1930, Institut Pasteur, 28, rue du Docteur-Roux, 75015 Paris, France. O.Danos : Généthon, Cnrs URA 1923, 1, rue Internationale, 91002 Evry Cedex, 'France. M. Peschanski : Inserm U.421/IM3, Faculté de médecine, 8, rue du Général-Sarrail, 94010 Créteil Cedex, France.

vent être définies aujourd'hui, et indispensable de le faire à plusieurs pour croiser les points de vue à partir d'expériences très différentes.

\section{Tendances actuelles...}

Les dix années passées ont été marquées par une exploration systématique des différents domaines dans lesquels un transfert de matériel génétique apparaissait comme une démarche thérapeutique possible. Ces travaux exploratoires ont notoirement éclairci la situation. Un regard plus pertinent a été porté sur la physiopathologie des affections candidates, permettant pour certaines d'entre elles de dresser un cahier des charges relativement précis, définissant les conditions qui autoriseraient des succès thérapeutiques par la thérapie génique. Cette exigence initialement formulée dans un rapport [1] fait au directeur du NIH (National Institutes of Health) en 1995 apparaît aujourd'hui essentielle, et I'on 
s'étonne déjà de la naïveté de certaines des directions qui ont été choisies durant ces dix dernières années. La conséquence la plus immédiate de cette évolution a été d'écarter un nombre notoire de pathologies préalablement supposées candidates pour une thérapie génique et de concentrer les efforts sur un nombre plus restreint de maladies apparaissant comme les plus favorables à une réussite. L'exigence $d^{\prime}$ une justification scientifique solidement argumentée sur une connaissance physiopathologique va en s'amplifiant au fil des années, et c'est probablement I'une des tendances les plus marquées, et des plus heureuses, de l'évolution récente de la discipline. La recherche d'outils adaptés a tenu une place prépondérante et reste le domaine le plus spécifique de la discipline. De nouveaux systèmes de transfert de gène ont été décrits à intervalles réguliers au cours des dernières années. Ils ont régulièrement été présentés comme la solution révolutionnaire et définitive à l'ensemble des problèmes liés à la thérapie génique... jusqu'à ce que leurs limitations deviennent trop évidentes pour être plus longtemps négligées, voire refoulées. L'appréciation des qualités effectives des outils proposés n'a en effet pas toujours bénéficié de toute I'objectivité souhaitable, et l'on peut se réjouir que progressivement s'estompe le fantasme du vecteur parfait. Bien que souvent chaotiques, ces avancées nous permettent toutefois aujourd'hui de dresser une sorte de catalogue des possibles, des souhaitables et des improbables. Ces clarifications quant au bon usage des vecteurs peuvent faire espérer qu'à l'exigence $d$ 'une meilleure compréhension des besoins spécifiques de chaque pathologie soit associée celle d'une meilleure pratique des outils disponibles, tel qu'il en résulte une conception plus réaliste d'éventuels traitements.

L'appréciation du risque associé aux manœuvres de transfert de gène a été longtemps largement négligée. Elle occupe le devant de la scène depuis les accidents survenus I'an passé aux États-Unis. La fuite en avant qui a justifié bon nombre des essais cliniques engagés au cours des cinq dernières années a de ce fait été brutalement ralentie. La prise en compte du danger potentiel pour les patients ne peut désormais plus être ignorée, de même que la nécessité d'une rigueur maximale dans la conception et dans la réalisation des protocoles. Ce qui est exigé de n'importe quelle approche innovante testée en clinique l'est à présent de la thérapie génique et il faut se réjouir de cet acte de reconnaissance. Comme ailleurs, I'appréciation réaliste du risque devient un élément majeur de décision, et les exigences en ce sens ne peuvent que s'accroître. Elles auront vraisemblablement un impact majeur sur le développement de la discipline. Les dispositifs réglementaires progressivement mis en place dans les pays concernés se voient ainsi conférer un rôle grandissant dans le choix des options de développement. Avec le recul disponible aujourd'hui, les instances de contrôle sont en mesure de définir un équilibre réaliste entre des attitudes trop laxistes ou trop restrictives. La multiplication de protocoles multicentriques internationaux met l'accent sur les disparités d'appréciation du risque entre les différents pays et devrait ainsi aider à la mise en place progressive d'une homogénéisation des réglementations. L'Union européenne a engagé des travaux en ce sens [2]. C'est aussi la préoccupation de chercheurs engagés dans des expérimentations cliniques, qui examinent la question dans le cadre du réseau Euregenethy [3].

Si l'ADN a acquis le statut de médicament, les biologistes moléculaires et les virologistes, qui sont les chercheurs majoritairement impliqués dans le développement de la thérapie génique, n'ont pas pour autant acquis celui de physiologiste ou de pharmacologiste. Leur formation explique probablement en partie certaines réticences observées vis-à-vis d'une expérimentation animale rigoureuse et une négligence évidente des questions liées à la biodisponibilité et à la pharmacocinétique des produits. L'industrie pharmaceutique, pourtant massivement présente dans la discipline, y apparaît le plus souvent sous la forme de petites entreprises de biotechnologie dont la culture scientifique est plus proche de celle du monde académique que de celle des grands pharmaciens. La possibilité de mettre effectivement sur le marché des produits de thérapie génique devrait conduire à une plus grande implication des professionnels de la pharmacie, et influer significativement sur les modes de raisonnement qui prévalent dans la discipline. Si cette tendance est bien visible en ce qui concerne les exigences vis-à-vis de la manufacture des produits, elle reste toutefois le plus souvent discrète en ce qui concerne la conception des stratégies et des outils.

Sous la pression des différents problèmes qui viennent d'être évoqués, on voit se dessiner une tendance générale de la thérapie génique vers une plus grande rationalisation: rationalisation des stratégies et des indications, rationalisation de l'appréciation du risque, rationalisation de la conception et de la manufacture des produits. Un corollaire possible de cette évolution pourrait être une inversion de la tendance actuelle à multiplier les essais thérapeutiques. Ceux-ci pourraient ainsi devenir moins nombreux, aux dépens de ceux dont les bases scientifiques sont les plus hypothétiques. Par voie de conséquence, I'accent mis jusqu'à présent sur les essais en cancérologie, qui concernent aujourd'hui plus de $70 \%$ des patients traités par des méthodes de transfert génétique [4], pourrait progressivement s'estomper.

\section{Directions futures...}

\section{La sécurité d'abord}

On peut tenir pour une certitude que les exigences de sécurité pour le patient et pour son environnement se feront chaque jour plus impératives. Les gestes à faible risque seront donc systématiquement favorisés, et l'orientation des recherches se fera en conséquence. Parmi les critères déterminant la sécurité du transfert, deux caractéristiques nous semblent prioritaires.

La première concerne la stabilité du transfert de gène. Une modification génétique réversible apparaîtra plus sûre qu'une modification définitive. Si le geste thérapeutique doit avoir un effet à long terme, la répétition d'un geste conduisant à une modification transitoire, pour autant que celui-ci soit peu invasif, sera préférée à un geste unique et définitif. Faute de 
pouvoir faire mieux, un contrôle strict de l'expression du transgène sera recherchée si le transfert se doit d'être stable. La seconde caractéristique importante pour la sécurité est le ciblage du transfert de gène. Une modification restreinte à la population cellulaire définie comme la cible thérapeutique sera éminemment préférable à une modification plus étendue, et a fortiori à une modification difficilement contrôlable. Si ces deux caractéristiques, réversibilité et ciblage du transfert de gène, devaient effectivement apparaître comme prioritaires, certaines tendances actuelles dans le développement de la thérapie génique pourraient s'en trouver modifiées, alors que d'autres se verraient confirmées.

\section{Qualité pharmaceutique}

Une première conséquence des exigences de sécurité serait un intérêt encore plus marqué qu'aujourd'hui pour le développement de vecteurs synthétiques, ou semi-synthétiques, destinés à être administrés in vivo, sur le mode d'un médicament. On entend par vecteurs semi-synthétiques des modifications chimiques de particules virales ou, à l'inverse, I'introduction de composants viraux dans des composés chimiques. On peut s'attendre au développement de nouveaux composés tels que lipoplexes ou polyplexes, et à la conception de préparations d'ADN plus "écologiques", en ce que les gènes de résistance aux antibiotiques en auront été retirés. La multiplication des essais thérapeutiques faisant appel à des injections uniques ou répétées d'ADN nu, éventuellement par électro-transfert, est vraisemblable dans des indications où un apport temporaire de la protéine thérapeutique est suffisant. La vaccination par ADN peut être rapprochée de cette situation.

Il est intéressant de noter que le caractère transitoire du transfert de gène, qui est aujourd'hui perçu comme une limitation des vecteurs synthétiques et conduit à rechercher la mise au point de systèmes intégratifs ou de chromosomes artificiels, apparaîtrait alors comme l'une de leurs propriétés intéressantes. Des améliorations décisives des vecteurs synthétiques pourraient en revanche concerner: I'efficacité du transfert, ce qui nécessite une meilleure connaissance de leur trafic dans la cellule; la possibilité d'une expression élevée, mais également contrôlable; le ciblage de populations cellulaires définies. La possibilité de restreindre la biodistribution, grâce par exemple à la fixation à un récepteur porté par la membrane cellulaire, apporterait un avantage supplémentaire à ces produits. La réparation de mutations par recombinaison in situ est potentiellement I'un des développements importants des vecteurs synthétiques. Du fait de leur nature synthétique et donc de la possibilité d'une manufacture industrielle classique, ces composés se rapprochent $d^{\prime}$ 'un médicament traditionnel, à la différence près que le principe actif est un acide nucléique. Leur production et leur évaluation peuvent être réalisés selon les normes de qualité de l'industrie pharmaceutique. On peut imaginer qu'ils trouvent, au fur et à mesure de leurs développements, une place dans la pharmacopée telle que leurs caractéristiques spécifiques de vecteurs de transfert de gène s'effacent progressivement au bénéfice de leurs propriétés de médicament.

\section{Efficacité virale}

Un transfert de gène stable restera nécessaire dans les nombreuses applications incompatibles avec l'administration répétée $d$ 'un vecteur synthétique. Dans ce cas, I'utilisation de vecteurs viraux restera probablement sans alternative encore longtemps.

Le choix doit alors se faire entre une administration du vecteur viral ex vivo ou in vivo. Ce choix est le plus souvent dicté aujourd'hui par la nature du tissu auquel le transfert de gène s'adresse. Ainsi, le transfert de gène dans les cellules hématopoïétiques est réalisé ex vivo alors que le transfert de gène dans le système nerveux se conçoit plus aisément au moyen d'une administration locale. Lorsque les deux types de manœuvre sont faisables, le choix fait intervenir une appréciation du risque et de I'efficacité. La question peut être posée aujourd'hui pour des tissus comme le foie, la paroi vasculaire, les articulations, ou pour la distribution de protéines thérapeutiques dans le sérum. Les critères de sécurité en rapport avec la réversibilité et le ciblage du transfert de gène arguent en faveur du transfert de gène ex vivo, suivi de la réimplantation de cellules génétiquement modifiées dans lesquelles il serait envisageable d'introduire un gène suicide. A l'inverse, les risques biologiques inhérents à la manipulation de cellules, qui peuvent être contaminées au cours de la culture par des pathogènes connus ou encore inconnus, tendraient à faire éviter l'étape ex vivo.

L'administration d'un vecteur viral in vivo apparaît souvent aujourd'hui comme la seule stratégie potentiellement efficace. En regard des bénéfices éventuels, le risque est en général considéré comme acceptable. II est probablement minime en ce qui concerne les vecteurs dérivés des virus associés aux adénovirus (AAV), si I'on met de côté le caractère irréversible du geste. L'administration chez l'homme de vecteurs dérivés de lentivirus rencontre plus de résistances, du fait du caractère hautement pathogène du VIH. C'est cependant I'appréciation du danger effectif qui justifiera probablement les premiers essais cliniques avec ces vecteurs dans des maladies pour lesquelles un bénéfice clinique est espéré. Le cerveau devrait être parmi les premières cibles envisagées. Après avoir été considéré comme le vecteur de choix dans un grand nombre de cas, I'adénovirus a été particulièrement discuté ces derniers temps, du fait des accidents qui sont survenus lors de nombreux essais. Ces accidents ont été bénins dans la plupart des cas, mais le décès d'un patient à Philadelphie (États-Unis) I'an dernier a eu un impact spectaculaire. Les recherches très actives qui portent spécifiquement sur les risques liés à ce vecteur depuis des années devront sans doute aboutir à l'établissement de règles d'utilisation strictes et validées pour que son développement clinique puisse reprendre.

Les vecteurs viraux considérés aujourd'hui comme porteurs d'un réel potentiel thérapeutique auront la difficile mission de valider ou d'invalider les stratégies thérapeutiques et les gènes candidats. La compréhension de la physiopathologie n'est jamais suffisante pour avoir I'assurance qu'un gène candidat sera effec- 
tivement thérapeutique. Les avancées dans l'étude des génomes devraient apporter un nombre grandissant de gènes potentiellement candidats, mais par ailleurs mal connus. La démonstration que l'expression d'une protéine donnée dans un tissu choisi apporte effectivement un bénéfice thérapeutique nécessite que le transfert de gène soit réalisé de la manière la plus efficace possible. II est bien évident que la situation idéale est celle où cette démonstration peut être apportée chez un modèle animal fidèle de la maladie humaine. Cette situation étant exceptionnelle, on peut concevoir que l'indispensable compromis entre risque et bénéfice favorise la mise en place d'essais cliniques reposant sur l'administration in vivo chez l'homme de vecteurs viraux au cours $d^{\prime}$ une phase initiale de démonstration de l'efficacité thérapeutique. Par exemple, la démonstration que des facteurs neurotrophiques ont un effet thérapeutique dans les maladies neurodégénératives nécessitera probablement des essais cliniques comportant un transfert de gène in situ dans le parenchyme cérébral. II est cependant vraisemblable que même en l'absence d'effet indésirable notoire, le caractère irréversible et non ciblé du transfert de gène réalisé avec ces vecteurs devienne difficilement acceptable dès lors que l'efficacité thérapeutique du gène candidat aura été démontrée. On peut imaginer que des méthodes alternatives, considérées comme plus sûres, se développent durant l'intervalle, modifiant de ce fait I'appréciation du rapport risque/bénéfice, et conduisant, en un deuxième temps, à renoncer à l'administration de vecteurs viraux directement in vivo.

\section{Réparation et reconstruction}

Des alternatives à la thérapie génique in vivo pourraient naître du développement de la thérapie cellulaire, lesquelles permettraient d'envisager plus fréquemment et dans de meilleures conditions un transfert de gène ex vivo. Deux notions d'importance inégale peuvent être prises en considération. D'abord, le développement de méthodes de culture en l'absence de sérum réduira considérablement le risque associé à la manipulation ex vivo de cellules destinées à être implantées chez un malade. Ensuite, et surtout, I'évolution de la thérapie cellulaire vers une discipline de plus en plus spécialisée dans la manipulation in vitro de cellules progénitrices pourrait rendre faisable la reconstruction de certains tissus à partir de cellules génétiquement modifiées, comme c'est le cas aujourd'hui pour le tissu hématopoïétique. Si I'option existe, il est vraisemblable qu'à la réparation in situ d'un tissu malade, on préférera sa reconstruction à partir de progéniteurs sains préalablement modifiés ex vivo par une thérapie génique. Le transfert de gène aurait pour objet de corriger un défaut génétique, de faire acquérir une nouvelle fonction, ou d'introduire un gène suicide permettant un contrôle du devenir de la population cellulaire qui a été manipulée ex vivo. II est vraisemblable que de telles méthodes seront prochainement évaluées en ce qui concerne les muscles squelettiques, le cœur et l'os. La tâche est plus ardue en ce qui concerne des tissus comme le foie, le pancréas ou le système nerveux central. Dans tous les cas, le développement de ces méthodes posent de multiples questions en regard de l'origine des cellules qui seront manipulées, de leur amplification et de leur caractérisation. La généralisation, en dehors de I'hématologie, de méthodes associant intimement un geste de thérapie génique à des manœuvres de thérapie cellulaire nécessitera la reconnaissance de marqueurs spécifiques, antigéniques ou génétiques, et la découverte de nouveaux facteurs de croissance afin d'apprendre à manipuler les cellules souches de différents tissus.

Quelles seront les places respectives des secteurs public et industriel dans ces développements attendus ? II est important de remarquer qu'une grande partie des recherches sur les cellules souches et leurs applications thérapeutiques se déroule dans le secteur industriel [5]. Ces activités d'amont, souvent menées par des entreprises de petite taille, sont encore éloignées des applications cliniques. Cependant, il est dès à présent souhaitable d'envisager et de favoriser la mise en place du traditionnel partage des compétences et des responsabilités par lequel les industriels s'efforcent de mettre à la disposition du milieu médical, des stratégies potentielles reposant sur de nouveaux outils (matériels pour l'isolement, la culture et I'identification des cellules d'intérêt), mais où il reviendra aux médecins hospitaliers d'en définir les indications thérapeutiques effectives. Cela suppose la mise en place d'équipes hospitalières multidisciplinaires spécialisées, associant intimement biologistes, pharmaciens et cliniciens pour la conception et la réalisation des essais cliniques, ainsi que l'information des médecins non spécialisés afin que de nouvelles stratégies thérapeutiques reposant sur ces méthodes soient imaginées.

\section{Conclusions}

Les auteurs qui ont contribué à cette discussion s'entendent à considérer qu'il serait tout aussi faux de nier aujourd'hui les perspectives ouvertes par la thérapie génique qu'il l'était, il y a dix ans, de rêver qu'elle allait révolutionner du jour au lendemain la pratique médicale. Le domaine subit incontestablement une maturation qui, certes, ne va pas sans heurts mais permet de mieux définir aujourd'hui sinon les voies précises de son développement, du moins certains grands axes qu'il va suivre. Parmi ceux-ci, comme nous l'avons souligné, il est intéressant de constater que la recherche en thérapie génique est manifestement en train d'évoluer en fonction de pressions d'ordre réglementaire, ou "sociétal», c'est-à-dire non directement scientifiques. La maturation du champ réglementaire, parallèle de celle du champ scientifique, permet aujourd'hui de mieux apprécier les vraies questions, les vraies limites, et donc les vrais besoins. Enfin, la thérapie génique est sans doute en train de pénétrer réellement le monde hospitalier, audelà des quelques centres de recherche pionniers, même si c'est encore par une petite porte ouverte sur quelques maladies rares et pas, comme on le rêvait il y a dix ans, par celle qui ouvrirait sur le cancer. L'heure est sans doute venue de préparer les premiers cadres nécessaires à ce passage du laboratoire vers l'hôpital 
RÉFÉRENCES

1. Marshall E. Less hype, more biology needed for gene therapy. Science 1995; 270: 1751. http://www.nih.gov/news/panelrep.html

2. http://biosafety.ihe.be/219cp/home.ncfk.

3. http://193.48.40.240//www/euregenethy/ euregenethy.html

4. http://www.wiley.co.uk/genetherapy/clinical

5. Marshall E. The business of stem cells. Science $2000 ; 287: 1419-21$.

TIRÉS À PART

J.M. Heard. $\mathbf{m} / \mathbf{S} 2000$

\section{Summary}

Where does gene therapy go?

Gene therapy, as well as the human genome project, expresses the implicit belief of our generation that improvement of human life supposes a better understanding of the mechanisms operating in living organisms. After 15 years of major endeavor, what has all this gene therapy work brought? which difficulties are we facing? how can we imagine the future of the field ? altogether, can we keep on with our belief? This paper reports discussions organized around those questions. For a more direct description of gene therapy studies, readers could refer to the special issue published by médecine/sciences in May 1999.

\section{5e colloque de la Société des Neuresciences TOULOUSE - $28-31$ mai 2001}

\section{Conférences plénières}

R. Hen (New York)

Modèle génétique d'anxiété et de dépression

F. Crépel (Paris)

Contrôle pré-synaptique de la plasticité synaptique des synapses glutamatergiques

P. Sokoloff (Paris)

Du clonage vers la clinique : I'exemple du récepteur D3 de la dopamine

M. Seagar (Marseille)

Canaux calcium et couplage excitation/exocytose dans les terminaisons nerveuses

Léon Tremblay (Paris)

Rôle de la motivation dans l'action : bases neuronales et comportementales chez le primate

F. Frackowiak (Londres)

The anatomy of brain function or the function of brain anatomy - system level studies in humans

J.A. Sahel (Strasbourg)

Signification en physiopathologie des interactions entre bâtonnets et cônes rétiniens

R. Dantzer (Bordeaux)

Mécanismes des effets comportementaux des cytokines

\section{Lecture Alfred Fessard}

N. Le Douarin (Nogent-sur-Marne)

La crête neurale, clé de l'évolution des vertébrés

12 Symposiums sur des thèmes divers

6 séances de communications affichées...

Date limite des inscriptions et de l'envoi des résumés de communications affichées 31 janvier 2001

Renseignements et inscriptions

Atout Organisation Science - 106, corniche Kennedy - 13007 Marseille

Téléphone : 0491527124 - Télécopie : 0491529373 - Messagerie : atoutsci@atout-org.com 